\title{
Open reduction and internal fixation of an osteochondral patellar fracture in an eleven year-old girl
}

\begin{abstract}
Osteochondral patellar fractures in pediatric age can result from acute patellar dislocations (APDs). The purpose of this article is to document and discuss the surgical treatment performed to a young female patient diagnosed with an osteochondral fracture (OCF) of the patella in the first episode of an APD.

We state the first episode of an APD in a 12 year-old girl, who presented with pain, edema and limited extension of the right knee, after a direct knee trauma. She had no predisposing factors regarding patellofemoral instability. This patient was treated at our Orthopaedic Surgery Department and then clinically (Kujala score) and radiographically evaluated, at 12 months of follow-up.

The knee radiograms gave rise to the suspicion of a large inferior osteochondral patellar fragment. Operative treatment was decided and open reduction and internal fixation (ORIF) of the fragment was performed, through a medial parapatellar approach. The fragment dimensions were $24 \times 18 \mathrm{~mm}$. The medial structures were addressed and repaired. The patient went through a rehabilitation programme and returned to full activities three months postoperatively. After 12 months of follow-up, there was full range of motion, no redislocation episodes and the Kujala score was 96.

Open reduction and anatomic in situ internal fixation of the osteochondral fragment was mandatory in this case, due to its dimensions and location. We consider this a safe and efficient technique that allows osteosynthesis of the osteochondral fragment; the approach used makes possible the acute repair of the medial structures. There is still no general consensus about whether or not to reconstruct the medial patellofemoral ligament (MPFL) in the context of a primary APD.
\end{abstract}

Keywords: Primary acute patellar dislocation; Osteochondral patellar fracture; ORIF; patellofemoral
Volume 6 Issue 2 - 2016

\author{
António Robalo Correia,' Nuno Marques \\ Luís, ${ }^{2}$ Igor Lima Martins,' ré Lopes Vasques,' \\ Filipa Gonçalves Pereira,' Francisco de Brito' \\ 'Department of Orthopaedic Surgery, Hospital Jose Joaquim \\ Fernandes, Portugal \\ ${ }^{2}$ Department of Orthopaedic Surgery, Clínica CUF Alvalade, \\ Portugal
}

Correspondence: Antonio Pedro Robalo Correia, Rua llidio Oliveira Guerra n35; 2430-463 Marinha Grande; Portugal, Tel 352000000000 Email aprobalo@gmail.com

Received: October 21, 2016 | Published: October 21, 2016

\section{Abbreviations}

APD, Acute Patellar Dislocation, OCF, Osteochondral Fracture, ORIF, Open Reduction Internal Fixation, MPFL, Medial Patellofemoral Ligament, ED, Emergency Department, ROM, Range Of Motion, VMO, Vastus Medialis Obliquous, MRI, Magnetic Resonance Imaging.

\section{Introduction}

Acute patellar dislocations (APD) are common in pediatric age and can be associated to osteochondral fractures (OCF) of the patella, the lateral femoral condyle, or both. ${ }^{1,2}$ According to some works. ${ }^{1,3}$ the incidence of OCF is underestimated. In fact, osteochondral patella fractures can be easily missed on plain knee radiograms. ${ }^{1,4}$ making neglected OCF of the patella a possible reason for claims for financial compensation against attending doctors. ${ }^{5}$ Although APD can occur even in the context of a stable knee, patient-related predisposing factors may contribute to dislocation. ${ }^{6,7}$ The goal of this article is to document and debate the surgical treatment performed to a patient with an OCF of the patella, in the context of the first episode of an $\mathrm{APD}$, in a previously stable patellofemoral joint.

\section{Case Presentation}

11 year-old healthy girl, with no previous symptoms of patellar instability, no known anatomic variations, nor hyperlaxity criteria. She was brought to the emergency department (ED) presenting with pain, edema and limited extension of the right knee, after a direct trauma of the patella- fall from her height, with the knee in flexion. Although she presented in the ED with a reduced patella, the story was compatible with patella dislocation and then reduction with an extension movement, performed by a local caregiver. The knee examination revealed a joint effusion under tension and the arthrocentesis revealed $70 \mathrm{~mm} 3$ of hematic liquid, with evidence of fat deposits. The tense knee hemarthrosis made us suspect of an OCF.

The knee radiograms revealed a free articular body that we interpreted as a possible osteochondral patella fracture (Figures 1 \& 2).

Due to the fragment characteristics (dimensions and location), surgical treatment was the option. In the same day, the child was operated: a medial parapatellar approach was chosen (Figure 3).

The intrarticular fragment was identified, measured $(24 \times 18 \mathrm{~mm})$, washed and cleaned (Figures 4 \& 5).

An open reduction and internal fixation with two subchondral $1.7 \mathrm{~mm}$ titanium alloy (Ti6A14V) headless screws was performed. Anatomic reduction was intraoperatively confirmed (Figure 6).

Since a medial arthrotomy was used, it was possible to suture the medial soft tissues, enhancing patellofemoral stability. The knee was then immobilized with a posterior protective cast for four weeks. 
After that period, she was immobilized with McConnell tapping and began a staged rehabilitation program: 1) Non-weight bearing exercises to gain range-of-motion (ROM); 2) Iliotibial band and hamstrings stretching; 3) Lateral patellar retinaculum stretching; 4) Gastrocnemius and quadriceps muscles stretching; 5) Quadriceps and vastus medialis obliquous (VMO) strengthening. Three months postoperatively, the patient was asymptomatic, with full ROM and already playing sports; the radiograms showed bone healing (Figures 7 and 8). At twelve months of follow-up, the Kujala score was 96 and there were no redislocation episodes.
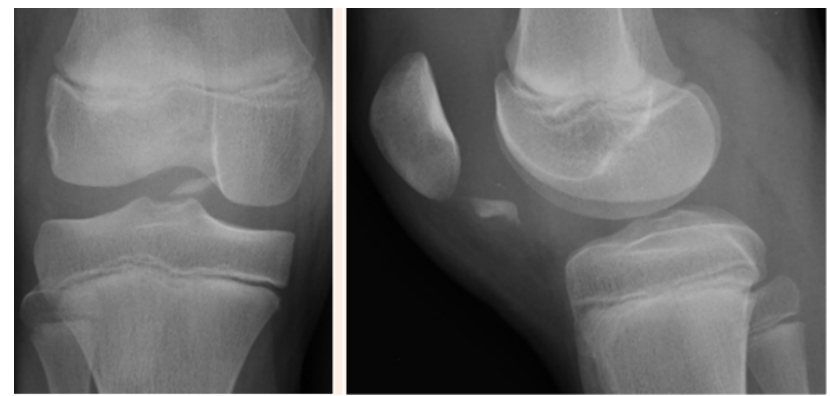

Figures I \& 2 AP and lateral radiograms: possible large inferior osteochondral patellar fragment, acting as a free articular body.

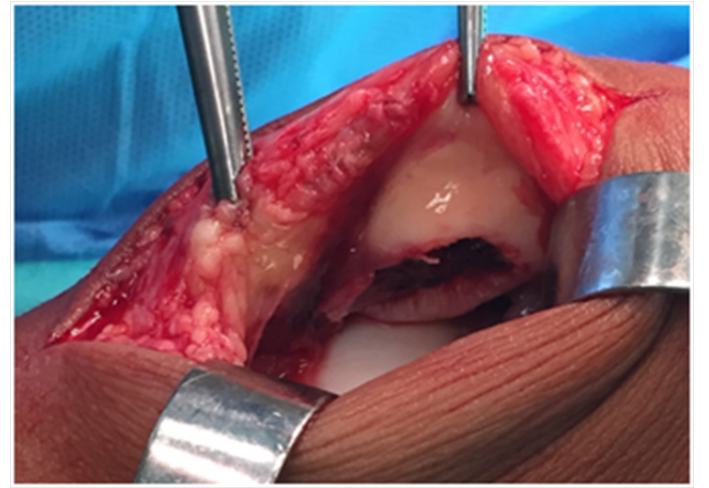

Figure 3 Medial parapatellar approach of the right knee, showing a large void beginning at the inferior pole of the patella, with extension to both facets, mostly the medial one.

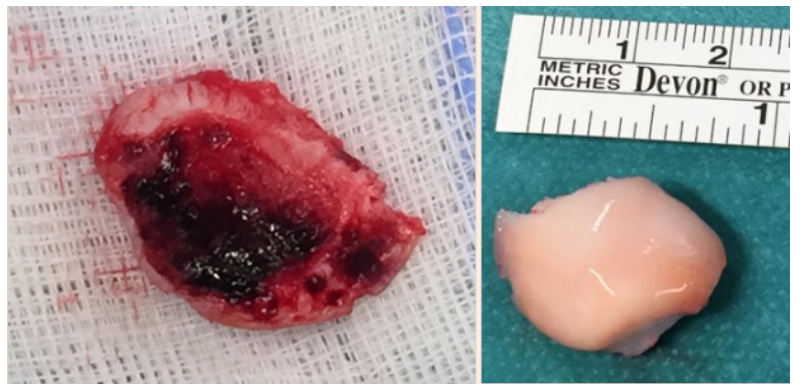

Figures 4 \& 5 Anterior and posterior faces of the osteochondral fragment.

\section{Discussion}

There are two main topics deserving discussion, suggested by this clinical case. The first one is about the diagnosis of this condition. In the context of an APD, the diagnosis of an OCF asks for a high degree of clinical suspicion and close look at the radiograms. In the presented case, the clinical data and the radiograms directed us to a possible diagnosis of a patellar OCF. A useful clinical clue was the presence of a tense hemarthrosis with fatty deposits when arthrocentesis was performed. However, it would have been wise to use magnetic resonance imaging (MRI) to enlighten the observed lesion, in what respects to certainty the source of the OCF (the patella), its dimensions and associated chondral alterations. ${ }^{8}$ Furthermore, MRI is of prime importance to localize possible medial patellofemoral ligament (MPFL) tears, which can be patellar, femoral or multifocal. In young children (aged 9 to 14), the patellar attachment site is the most commonly damaged, occurring a total rupture in more than half of the cases. ${ }^{9}$

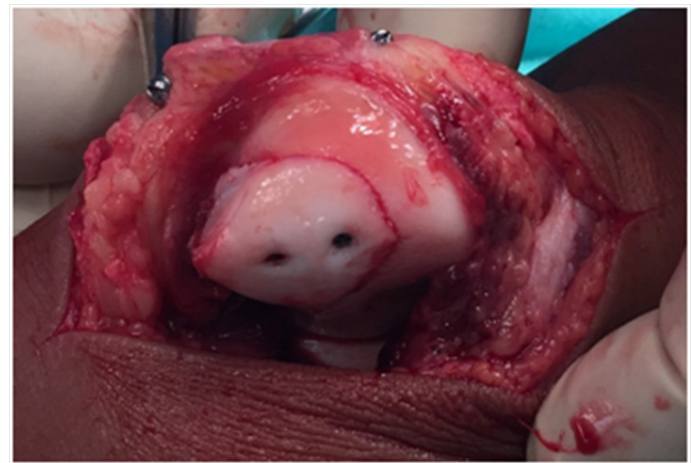

Figure 6 Anatomic reduction of the patella, with two $1.7 \mathrm{~mm}$ screws, subchondrally inserted.

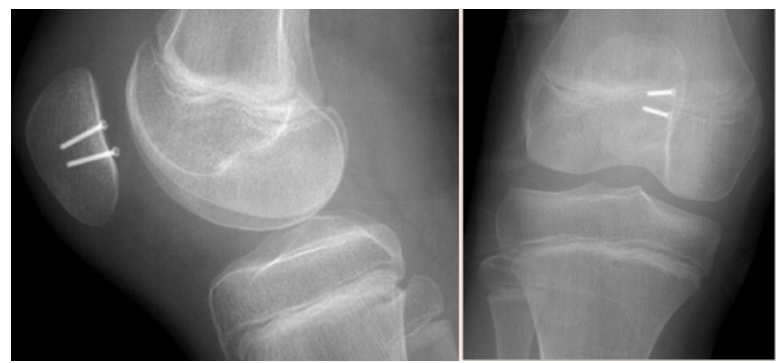

Figures 7 \& 8 Postoperative radiograms (3 months), showing good placement of the $1.7 \mathrm{~mm}$ screws and bone healing.

The second topic prone to discussion refers to treatment. Considering the initial step consensual towards surgical treatment, four major questions emerged next: 1) Which approach to use? 2) How to fix the osteochondral fragment? 3) Conservative versus repair versus reconstruction of the medial structures; 4) Which physiotherapy program to adopt postoperatively? Concerning the first question, the medial parapatellar approach used was related to the osteochondral fragment characteristics, whose majority belonged to the medial patellar facet. In this particular case, there was no indication for lateral patellar retinaculum release. About fragment fixation, the articular character of the fracture demanded an anatomic reduction, with absolute stability. The option between fixing and excising the fragment related to the fragment size $(24 \times 18 \mathrm{~mm}$, amenable to fixation). An otherwise small OCF, if symptomatic, would have been excised. ${ }^{7}$ Despite representing a last resort procedure, excision combined with microfractures grants satisfactory results, according to some studies. ${ }^{10}$ We opted for internal fixation with two headless $1.7 \mathrm{~mm}$ titanium alloy screws. Other viable option would have been biodegradable implants, but some important side effects have been demonstrated. ${ }^{11}$ ${ }^{12}$ In what concerns to the action taken towards the MPFL, arguments for conservative treatment are: this was the first episode of an APD. ${ }^{13}$ there were no anatomic variations, nor hyperlaxity criteria (MPFL rupture is not the cause, but a consequence of an APD). The arguments for surgical treatment, whether repair or reconstruction, are: since a medial arthrotomy was used, a MPFL repair would be a reasonable procedure; some prospective studies begin to show better long term results for MPFL reconstruction, compared to those observed with 
conservative treatment. ${ }^{14}$ As our knowledge about the biomechanical importance of the MPFL evolves - mainly its role against lateral displacement during motion and under high lateral stresses. ${ }^{15}$ - the more orthopaedic surgeons consider MPFL reconstruction the mainstay of treatment, even in the context of a primary APD. ${ }^{16}$ Postoperatively, the McConnell tapping method was applied, with the goals of protecting and maintaining / improving the patellofemoral biomechanics. ${ }^{17}$ In summary, despite paucity of diagnostic imaging exams, a good functional outcome was achieved. We believe that was due to a wellchosen and performed surgical technique and a correct postsurgical rehabilitation programme. Despite all the available evidence and progresses made towards a progressively better treatment for these patients, there is still no universal true about the perfect treatment for patients suffering from primary APD that can comprise all the specter of possible clinical variations. ${ }^{18}$

\section{Acknowledgments}

None.

\section{Conflicts of interest}

None.

\section{References}

1. Seeley MA, Knesek M, Vanderhave KL Osteochondral injury after acute patellar dislocation in children and adolescents. J Ped Orthop. 2013:33(5):511-518.

2. Jalan D, Morey VM, Mittal R et al. Transient Patellar Dislocation Resulting in Simultaneous Osteochondral Fractures of Patella and Lateral Femoral Condyle- A Case Report. J ClinDiagn Res. 2014:8(10):LD04 LD06.

3. Stanitski CL, Paletta GA Articular cartilage injury with acute patellar dislocation in adolescents. Arthroscopic and radiographic correlation. Am J Sports Med. 1998:26(1):52-55.

4. Enea D, Busilacchi A, Cecconi $\mathrm{S}$ et al. Late-diagnosed large osteochondral fracture of the lateral femoral condyle in an adolescent: a case report. J Ped Orthop B. 2013:22(4):344-349.

5. Leeberg V, Sonne-Holm S, Christoffersen JK et al. Fractures of the knee in children-what can go wrong? A case file study of closed claims in The Patient Compensation Association covering 16 years. J Child Orthop 2015:9(5):391-396.

6. Dejour H, Walch G, Nove-Josserand L et al Factors of patellar instability: An anatomic radiographic study. Knee Surg, Sports traumatol, Arthroscopy. 1994:2(1):19-26.
7. Mulford JS, Wakeley CJ, Eldridge JDJ Assessment and management of chronic patellofemoral instability. $J$ Bone Joint Surg.Series B. 2007:89(6):709-716.

8. Engelhardt LV, Raddatz M, Bouillon B et al. How reliable is MRI in diagnosing cartilaginous lesions in patients with first and recurrent lateral patellar dislocations? BMC Musculoskelet Disord. 2010:11:149.

9. Askenberger M, Arendt EA, Ekstrom W et al. Medial Patellofemoral Ligament Injuries in Children With First-Time Lateral Patellar Dislocations: A Magnetic Resonance Imaging and Arthroscopic Study. Am J Sports Med. 2016:44(1):152-158.

10. Lee BJ, Christino MA, Daniels AH et al. Adolescent patellar osteochondral fracture following patellar dislocation. Knee Surg, Sports traumatol, Arthroscopy. 2013:21(8):1856-1861.

11. Barfod G, Svendsen RN Synovitis of the knee after intraarticular fracture fixation with Biofix. Report of two cases.Acta Orthop Scand. 1992:63(6):680- 681 .

12. Friden $T$, Rydholm $U$ Severe aseptic synovitis of the knee after biodegradable internal fixation: a case report. Acta Orthop Scand. 1992:63(1):94-97.

13. Nikku R, Nietosvaara $Y$, Aalto $K$ et al. Operative treatment of primary patellar dislocation does not improve medium-term outcome: a 7-year follow-up report and risk analysis of 127 randomized patients. Acta Orthop. 2005:76:699-704.

14. Camanho G, Viegas Ade C, Bitar AC et al.Conservative versus surgical treatment for repair of the medial patellofemoral ligament in acute dislocations of the patella. Arthroscopy. 2009:25(6):620-625.

15. Zaffagnini $\mathrm{S}$, Colle $\mathrm{F}$, Lopomo $\mathrm{N}$ et al The influence of medial patellofemoral ligament on patellofemoral joint kinematics and patellar stability. Knee Surg, Sports traumatol, Arthroscopy. 2009:21(9):2164-2171.

16. Maffulli N, Via AG,Oliva F Reconstruction of the Medial Patellofemoral Ligament: A Surgical Technique Perspective from an Orthopedic Surgeon. Sports Injuries. 2015:1409-1415.

17. Chang WD, Chen FC, Lee CL et al. Effects of KinesioTaping versusMcConnell Tapingfor Patellofemoral Pain Syndrome: A Systematic Review and Meta-Analysis. Evid Based Complement Alternat Med 2015:471208.

18. Coviello M, Mazzola C Management of the First Patellar Dislocation. In: Volpi (Ed.), Arthroscopy and Sport Injuries, Springer, USA, pp. 2016:393-397. 